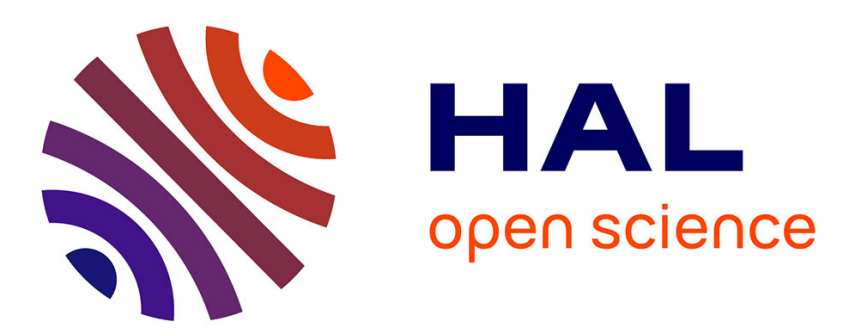

\title{
Caractérisation par A. T. D. de verres du système Se-Te-Ge0,5 Sb0,5
}

S. Bordas, M. D. Baró, J. Casas-Vazquez, N. Clavaguera, M. T.

Clavaguera-Mora

\section{- To cite this version:}

S. Bordas, M. D. Baró, J. Casas-Vazquez, N. Clavaguera, M. T. Clavaguera-Mora. Caractérisation par A. T. D. de verres du système Se-Te-Ge0,5 Sb0,5. Revue de Physique Appliquée, 1977, 12 (5), pp.681-685. 10.1051/rphysap:01977001205068100 . jpa-00244225

\section{HAL Id: jpa-00244225 https://hal.science/jpa-00244225}

Submitted on 1 Jan 1977

HAL is a multi-disciplinary open access archive for the deposit and dissemination of scientific research documents, whether they are published or not. The documents may come from teaching and research institutions in France or abroad, or from public or private research centers.
L'archive ouverte pluridisciplinaire HAL, est destinée au dépôt et à la diffusion de documents scientifiques de niveau recherche, publiés ou non, émanant des établissements d'enseignement et de recherche français ou étrangers, des laboratoires publics ou privés. 


\title{
CARACTÉRISATION PAR A. T. D. DE VERRES DU SYSTÈME Se-Te-Ge ${ }_{0,5} \mathbf{S b}_{0,5}$
}

\author{
S. BORDAS, M. D. BARÓ, J. CASAS-VÁZQUEZ \\ Departamento de Termología, Universidad Autónoma de Barcelona, Bellaterra, Espagne \\ N. CLAVAGUERA \\ Facultad de Física, Universidad de Barcelona, Espagne \\ M. T. CLAVAGUERA-MORA \\ Departamento de Física Fundamental, Universidad de Valladolid, Espagne
}

\begin{abstract}
Résumé. - La plus grande difficulté qui apparaît quand on essaye d'étudier les propriétés des verres semiconducteurs est due à leur caractère métastable. Ainsi la connaissance et le contrôle de la méthode de préparation est indispensable, car le traitement thermique modifie fréquemment de manière drastique les propriétés du solide obtenu. Nous présentons le domaine de formation de verres obtenus par refroidissement du mélange liquide pour différentes conditions (vitesses comprises entre $10^{-3}{ }^{\circ} \mathrm{C} / \mathrm{s}-10^{2}{ }^{\circ} \mathrm{C} / \mathrm{s}$ ) ou, ce qui revient au même, la vitesse de refroidissement critique pour un grand nombre de compositions du système Se-Te-Ge $\mathrm{Ge}_{0,5} \mathrm{Sb}_{0,5}$. Pour déterminer les limites de la méthode d'ATD on compare les thermogrammes obtenus pour un même échantillon à différentes vitesses de chauffe et la continuité de la variation du thermogramme en fonction de la composition pour une vitesse de chauffe fixée. Cette étude permet en plus d'obtenir des approches de la cinétique de cristallisation et la continuité de variation des propriétés en fonction de la composition.

Abstract. - In the study of glassy semiconductors properties, there is an important difficulty arising from their metastable character. Thus the knowledge and control of the preparation method is essential, because the properties of the obtained solid may change drastically with their thermal history. In the present work the glass-forming region of the $\mathrm{Se}-\mathrm{Te}-\mathrm{Ge}_{0.5} \mathrm{Sb}_{0.5}$ system for different quenching conditions (cooling rates beetwen $10^{-3}{ }^{\circ} \mathrm{C} / \mathrm{s}$ and $10^{2}{ }^{\circ} \mathrm{C} / \mathrm{s}$ ) is reported, and the critical cooling rates for various compositions are determined. The limitations of the DTA method are shown when comparing, for a fixed composition, the influence of heating rate on the thermograms, and the continuity of variation of the thermograms with composition, for a fixed heating rate. This work allows also to get some insight both on crystallization kinetics and on the continuous variation of the physical properties with composition.
\end{abstract}

1. Introduction. - Contrairement aux solides cristallins, l'existence d'un verre n'est pas limitée par des questions de stœchiométrie mais seulement par la possibilité de réaliser une trempe assez rapidement pour empêcher que se produise la cristallisation. Ainsi on a pu mettre en évidence que certains verres chalcogénures présentent des propriétés physiques qui varient légèrement avec la composition [1], ce qui permet en principe de prévoir un alliage de caractéristiques convenables pour une application technique déterminée.

On a donc une grande liberté de choix quant à la composition du verre, mais il reste alors une grave difficulté pour l'étude de ses propriétés qui est dûe à son caractère métastable. L'étude des transformations propres à l'état vitreux est, par ce fait, un des moyens de caractérisation des verres. Depuis longtemps on connaît une loi empirique qui détermine qu'un matériau est bon formateur de verre s'il satisfait la relation:

$$
T_{\mathrm{g}} / T_{\mathrm{m}}=2 / 3
$$

où $T_{\mathrm{g}}$ est la température de la transition vitreuse et $T_{\mathrm{m}}$ la température de fusion. Dans le cas des verres chalcogénures Hruby [2] a introduit le paramètre $K_{\mathrm{gl}}$ pour caractériser l'aptitude à la vitrification qui est donné par

$$
K_{\mathrm{gl}}=\frac{T_{\mathrm{r}}-T_{\mathrm{g}}}{T_{\mathrm{m}}-T_{\mathrm{r}}}
$$

où $T_{\mathrm{r}}$ est la température de recristallisation. Suivant cet auteur un matériel est bon formateur de verre si son $K_{\mathrm{gl}}$ est supérieur à 0,1 .

Nous allons montrer quelle est la nature de l'information que peut fournir l'ATD pour la caractérisation des verres en nous appuyant sur les thermogrammes obtenus pour des échantillons situés sur le plan d'égale composition en $\mathrm{Ge}$ et $\mathrm{Sb}$ du système quaternaire $\mathrm{Se}-\mathrm{Te}-\mathrm{Ge}-\mathrm{Sb}$ qui est représenté sur la figure 1. Cette information permet premièrement d'obtenir le domaine de formation de verres, dont nous présenterons les avantages et inconvénients. Deuxiè- 
mement elle conduit à montrer la continuité de la variation des propriétés des verres, étude que nous réalisons plus particulièrement sur la ligne $\mathrm{Se}_{66,6-x} \mathrm{Te}_{x} \mathrm{Ge}_{16,6} \mathrm{Sb}_{16,6}$ (représentée en tracé continu sur la figure 1). Par ailleurs elle suggère l'existence

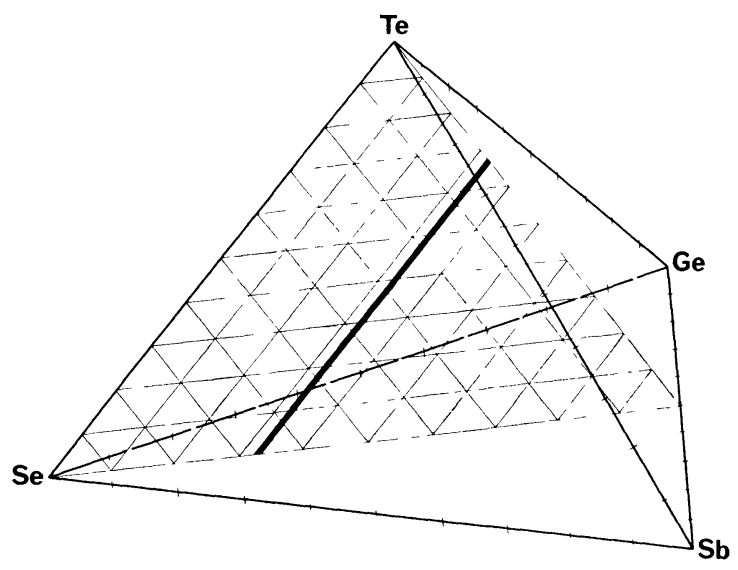

FIG. 1. - Domaine de compositions étudié.

de limites à l'utilisation du paramètre $k_{\mathrm{gl}}$, limites qui sont mises en évidence et dont nous essayons de nous affranchir. Finalement l'analyse de l'influence de la vitesse de chauffe dans une étude par ATD nous permet, en outre, d'obtenir des approches à la cinétique de cristallisation.

2. Domaine de formation de verres. - Nous avons déterminé le domaine de formation de verres par trempe à l'air et par refroidissement du mélange liquide à $5^{\circ} \mathrm{C} / \mathrm{min}$. Pour cela nous avons préparé et analysé les échantillons avec les méthodes décrites précédemment [3]. Nous présentons les résultats sur les figures $2 a$ et $2 b$. Sur ces figures nous avons représenté en noir les régions correspondant aux échantillons vitreux qui ne présentent pas de recristallisation au cours d'une chauffe à $5^{\circ} \mathrm{C} / \mathrm{min}$, et en gris celles des échantillons que, tout en étant non cristallins après obtention, recristallisent pour une vitesse de chauffe de $5^{\circ} \mathrm{C} / \mathrm{min}$. Les régions hachurées correspondent aux échantillons partiellement cristallins.

Un des avantages de l'ATD pour la caractérisation des verres est la facilité avec laquelle on obtient la stabilité des verres, que l'on peut mesurer par l'existence et la valeur de la température de recristallisation. C'est pour cette raison que nous avons différencié les verres qui recristallisent par chauffe de ceux qui passent directement à l'état liquide. Néanmoins un des inconvénients de l'ATD réside dans le fait qu'il est préférable de préparer les substances en poudre, et de ce fait la recristallisation se produit à des températures plus basses. Si l'on compare les figures $2 a$ et $2 b$ l'on voit que la région en noir est beaucoup plus étendue pour les échantillons obtenus après refroidissement à $5^{\circ} \mathrm{C} / \mathrm{min}$ pour ceux obtenus par trempe. Ceci est dû au fait que sur la figure $2 a$ nous présentons
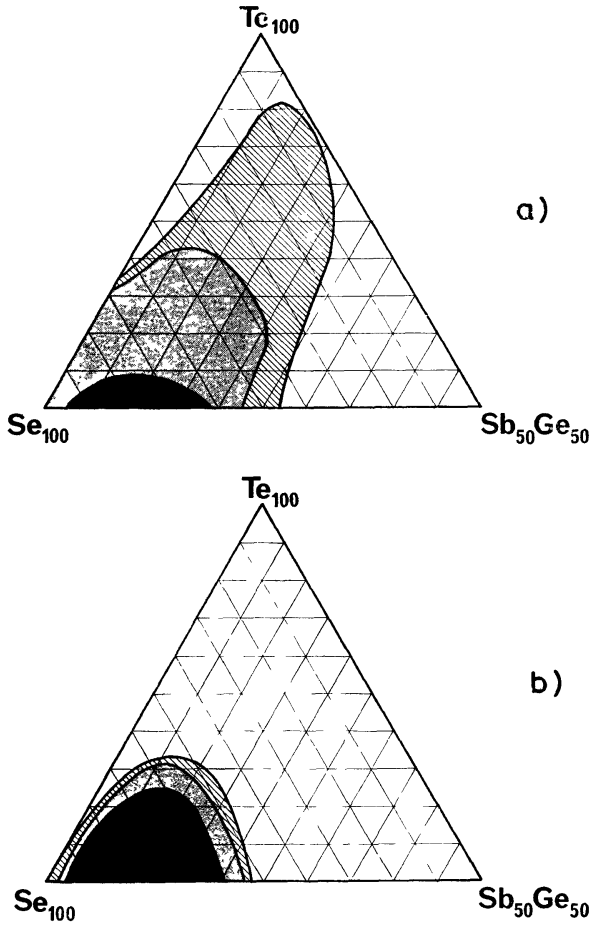

FIG. 2. - Domaine de formation de verres. $a$ ) par trempe à l'air ; $b$ ) après refroidissement à $5^{\circ} \mathrm{C} / \mathrm{min}$.

les résultats obtenus par ATD sur des échantillons en poudre, tandis que sur la figure $2 b$ il s'agit de résultats sur des échantillons massiques.

Puisque les limites du domaine de formation de verre varient avec la méthode de préparation, on attache généralement une grande importance à la vitesse de refroidissement critique au-delà de laquelle l'échantillon est non cristallin. Ce paramètre caractérise aussi la stabilité du verre, et peut être obtenu expérimentalement du côté des faibles vitesses par ATD et pour des vitesses plus élevées par trempe contrôlée. Nous présentons sur la figure 3 la répartition obtenue des vitesses de refroidissement critique en fonction de la

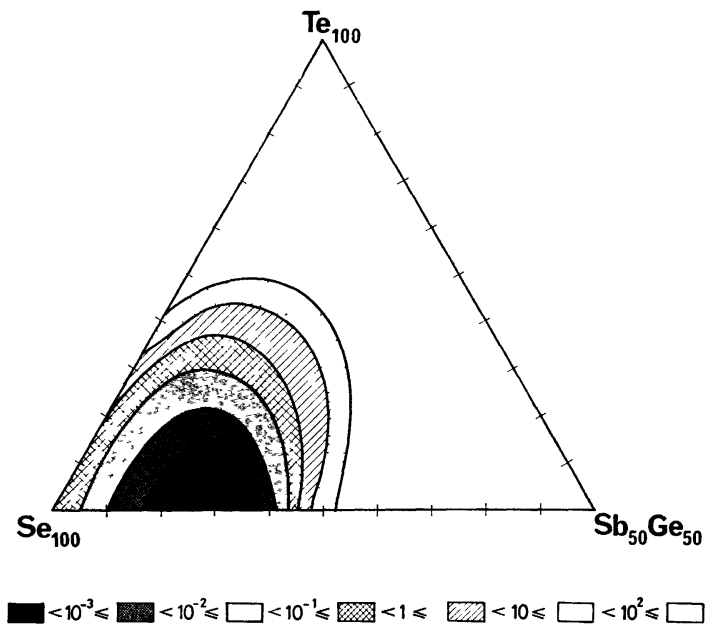

Fig. 3. - Répartition des vitesses de refroidissement critique en ${ }^{\circ} \mathrm{C} / \mathrm{s}$. 
composition pour des valeurs comprises entre $10^{-3} \mathrm{o} / \mathrm{s}$ et $10^{2} \mathrm{C} / \mathrm{s}$ pour le système $\mathrm{Se}-\mathrm{Te}-\mathrm{Ge}_{0,5} \mathrm{Sb}_{0,5}$.

Un des avantages de l'obtention du domaine de formation de verres réside dans la possibilité de pouvoir représenter, d'une manière simple, le mécanisme de formation de verre en partant de l'idée que le motif de base d'un verre est une unité de structure comprenant un atome et ses plus proches voisins [4]. Dans notre cas le domaine vitreux s'appuie d'un côté sur le système $\mathrm{Se}-\mathrm{Ge}_{0,5} \mathrm{Sb}_{0,5}$ où l'on admet [4] l'existence des unités de structure $\mathrm{Se}_{-} \mathrm{Se}_{2 / 2}$ et $\mathrm{GeSe}_{4 / 2}$ réunies en une double chaîne et l'insertion du $\mathrm{Sb}$ se fait par substitution au sélénium par deux atomes à la fois, ce qui aboutit à la condition $\mathrm{Se} \geqslant 2 \mathrm{Ge}$. Les résultats présentés sur la figure 2 suggèrent que l'insertion du quatrième élément $\mathrm{Te}$ donne lieu à l'apparition des unités $\mathrm{TeSe}_{2 / 2}$ et $\mathrm{GeTe}_{4 / 2}$ sujettes à la condition $\mathrm{Se} \geqslant 2 \mathrm{Te}$ pour que les liaisons Te-Te soient statistiquement peu probables.

Un autre avantage du domaine de formation de verre est de réunir en un seul diagramme, de manière qualitative, la stabilité des verres obtenus avec des méthodes contrôlées. La connaissance de la vitesse de refroidissement critique et aussi des températures de début et fin de cristallisation, pour une vitesse d'analyse donnée, permet de donner l'allure de la courbe de transformation temps-température (TTT) [5]. Nous présentons sur la figure 4 la courbe TTT pour un

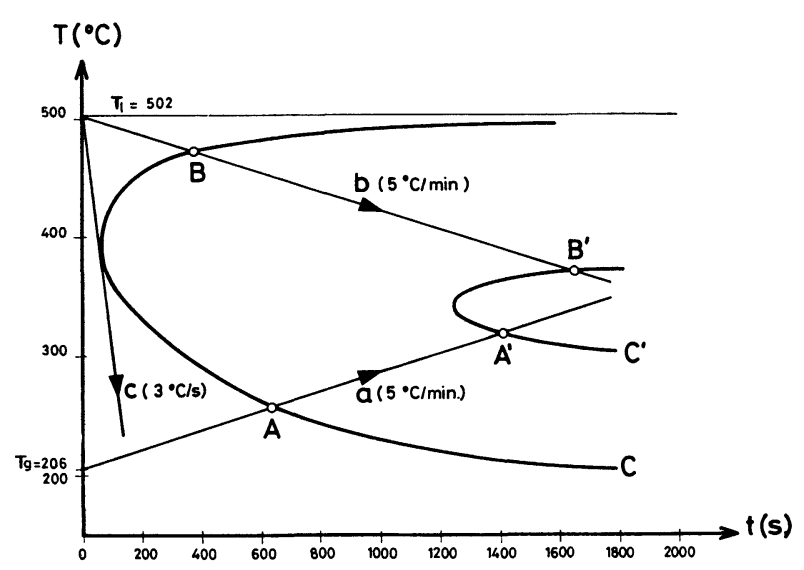

FIG. 4. - Courbe de transformation temps-température pour le $\mathrm{Se}_{41,6} \mathrm{Te}_{25} \mathrm{Ge}_{16,7} \mathrm{Sb}_{16,7}$.

échantillon de $\mathrm{Se}_{41,6} \mathrm{Te}_{25} \mathrm{Ge}_{16,7} \mathrm{Sb}_{16,7}$ tracée à partir des points expérimentaux $\mathrm{A}, \mathrm{A}^{\prime}, \mathrm{B}, \mathrm{B}^{\prime}$ et de la ligne qui correspond à la vitesse de refroidissement critique $c=3^{\circ} \mathrm{C} / \mathrm{s}$. La courbe $\mathrm{C}$ doit être tangente à cette ligne et représente le lieu géométrique des points où la cristallisation commence, tandis qu'après la courbe $\mathrm{C}^{\prime}$ l'échantillon est totalement cristallin.

3. Continuité de la variation des propriétés du verre. - L'étude réalisée du domaine de formation de verres nous permet d'obtenir une information qualitative sur la stabilité des verres en fonction de la composition. Nous présentons maintenant une étude plus poussée réalisée sur la ligne

$$
\mathrm{Se}_{66,6-x} \mathrm{Te}_{x} \mathrm{Ge}_{16,7} \mathrm{Sb}_{16,7}
$$

du diagramme qui traverse différentes régions du domaine de formation de verres. Nous portons sur la figure 5 les thermogrammes obtenus pour $0 \leqslant x \leqslant 29,2$. Pour une faible concentration de $\mathrm{Te}$ on observe seulement la transition vitreuse. Des concentrations plus élevés en Te montrent aussi la recristallisation (vraisemblablement $\mathrm{Te}$ et $\mathrm{GeTe}$ ) qui est suivie de la fusion.

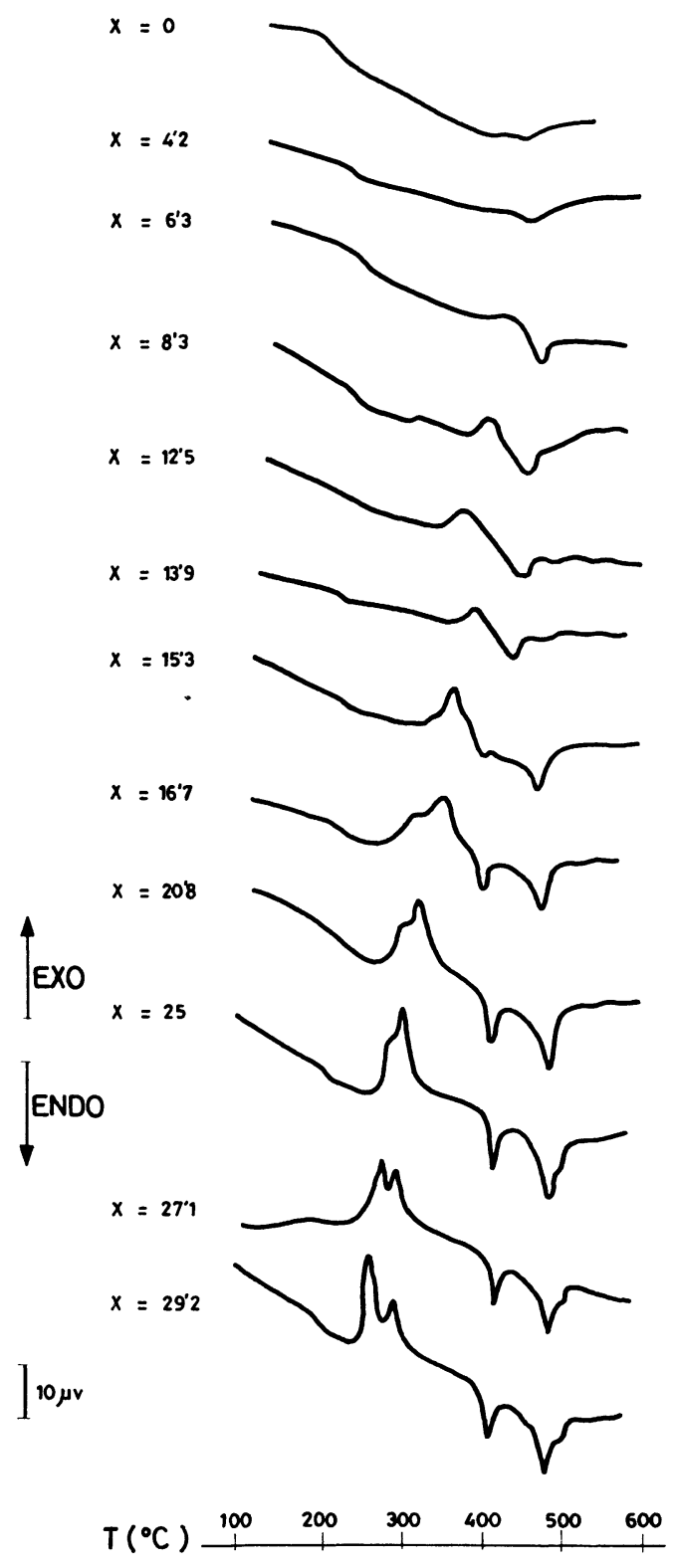

FIG. 5. - Thermogrammes des compositions

$\mathrm{Se}_{66,6-x} \mathrm{Te}_{x} \mathrm{Ge}_{16,7} \mathrm{Sb}_{16, \cdot 7}$

D'une manière générale le passage à travers des frontières de vitrification a lieu avec un changement continu des propriétés thermiques des échantillons et les thermogrammes de la figure 5 sont un 
exemple du comportement observé pour le système $\mathrm{Se}-\mathrm{Te}-\mathrm{Ge}_{0,5} \mathrm{Sb}_{0,5}$. Cette figure suggère aussi d'autres commentaires. Tout d'abord quand nous avons une ligne de base qui ne présente de déplacements dus ni à la recristallisation ni à la fusion nous ne pouvons pas conclure à l'inexistence de la recristallisation car probablement la recristallisation et la fusion sont alors simultanées. Dans l'exemple cité on peut porter les températures du début et de la fin de transformation en fonction du contenu en tellure (Fig. 6) et on constate qu'au fur et à mesure que le contenu en $\mathrm{Te}$ diminue, la recristallisation et la fusion se rapprochent jusqu'à se superposer.

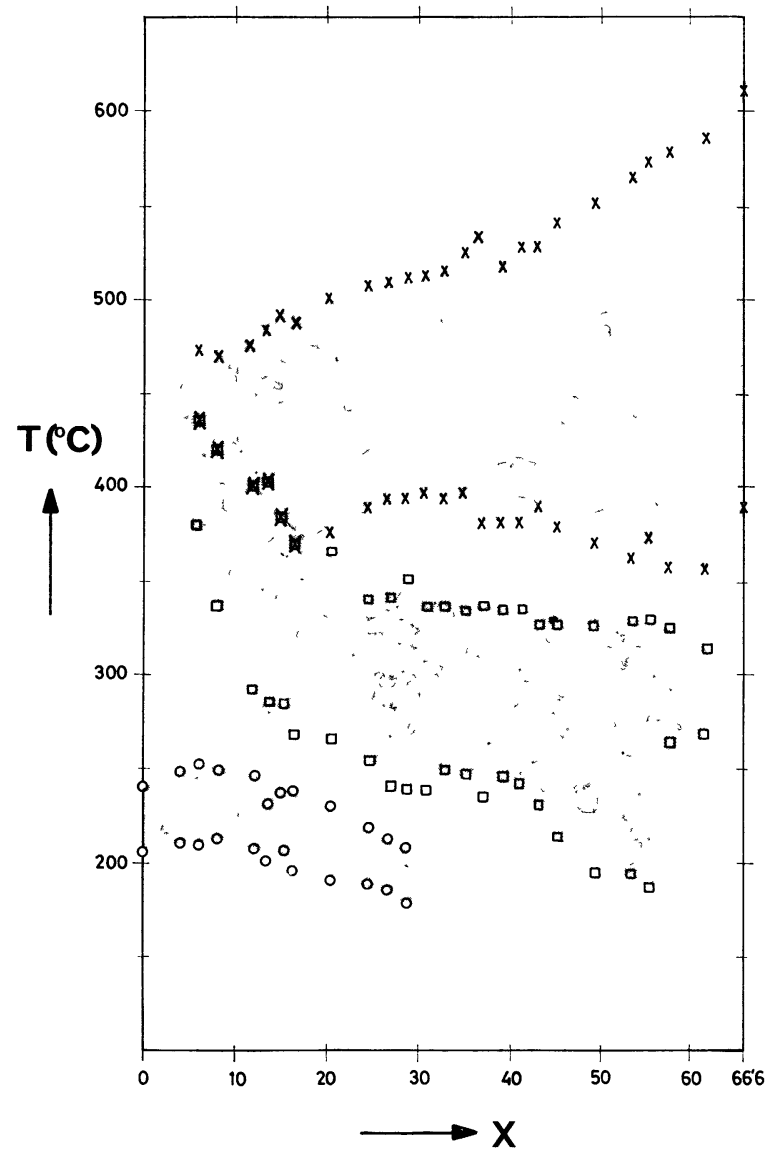

Fig. 6. - Températures du début et de la fin des transformations en fonction du contenu en tellure : $\bigcirc$ transition vitreuse ; $\square$ recristallisation; $\times$ fusion.

Par ailleurs dans l'intervalle des compositions où la recristallisation et la fusion se chevauchent la température de solidus reste mal définie ce qui rend difficile l'obtention du paramètre $K_{\mathrm{gl}}$. Ainsi, pour caractériser l'aptitude à la vitrification il serait préférable de remplacer $T_{\mathrm{m}}$ par $T_{\text {liquidus }}$ dans l'expression de $K_{\mathrm{gl}}$. Ceci aurait un autre avantage qui vient du fait que la température de solidus - contrairement à celle de liquidus - peut varier brusquement d'une composition à une autre très proche par suite de la nature du diagramme de phases ce qui rend difficile l'interprétation des valeurs de $K_{\mathrm{gl}}$ obtenues.
4. Analyse de l'influence de la vitesse de chauffe. Nous avons discuté jusqu'ici l'aptitude à la formation et la stabilité des verres à partir principalement de l'allure des thermogrammes obtenus pour une vitesse d'analyse donnée. Il est indispensable d'analyser toutes les compositions avec la même vitesse de chauffe si nous voulons comparer le comportement des différents alliages. Mais pour se rendre compte des limites de la méthode exposée il est nécessaire aussi d'étudier l'influence de la vitesse de chauffe sur les thermogrammes obtenus. La figure 7 montre, pour un même

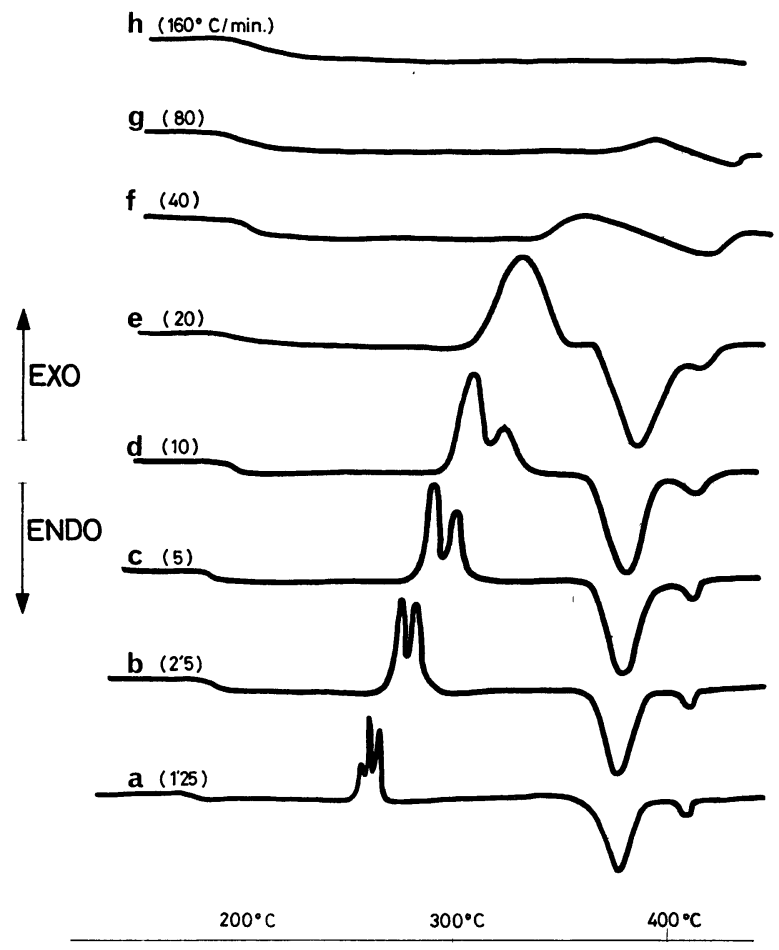

FIG. 7. - Courbes du calorimètre différentiel à balayage en fonction de la vitesse de chauffe.

échantillon, les thermogrammes obtenus à différentes vitesses de chauffe, sur un calorimètre différentiel à balayage Perkin Elmer. On peut interpréter ces résultats avec un diagramme enthalpie

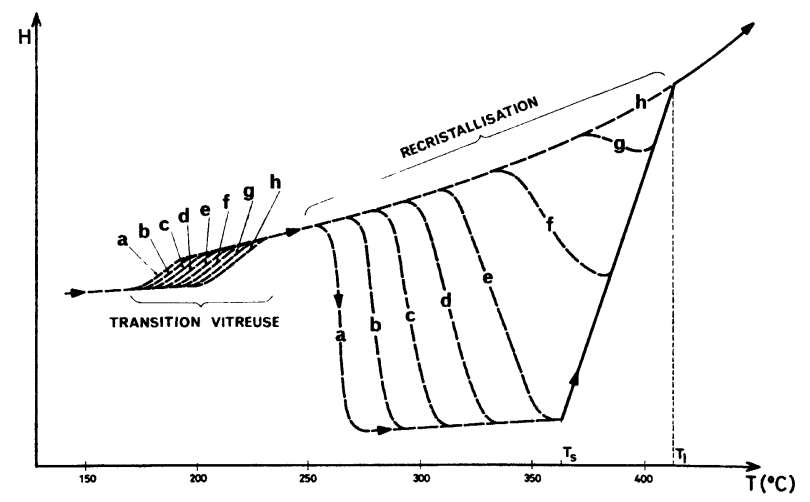

FIG. 8. - Représentation schématique des transformations du verre en fonction de la température et de la vitesse de chauffe. 
température (Fig. 8) en traçant les chemins hypothétiques parcourus dans les différentes expériences. On observe que la température de transition vitreuse et surtout celle de recristallisation se déplacent vers des valeurs plus faibles quand la vitesse de chauffe diminue. Il faut remarquer aussi le dédoublement progressif du pic de recristallisation en deux et même trois pics pour les faibles vitesses.

Une étude de la cinétique de cristallisation ne pourra donc pas s'appuyer seulement sur des données thermiques mais devra se compléter par des études microscopiques (rayons $\mathrm{X}$, microscopie électronique à balayage, etc.) pour identifier les différentes phases de la recristallisation.

Pour conclure nous devons signaler que la comparaison des figures 5 et 7 montre à quel point les limites du domaine de formation de verres qui ne se recristallisent pas par chauffe varient avec la vitesse d'analyse utilisée. Ce résultat n'est pas surprenant car la vitesse de chauffe est un paramètre essentiel dans la cinétique de cristallisation non isotherme. C'est pour cette raison qu'une telle classification est valable seulement si toutes les compositions sont analysées à la même vitesse de chauffe.

\section{Bibliographie}

[1] Mott, N. F. and Davis, E. A., Electronic Processes in NonCrystalline Materials (Oxford Univ. Press) 1971.

[2] Hruby, A., Czech. J. Phys. B 22 (1972) 1187.

[3] BoRDAS, S. et al., Journées d'Etude sur la caractérisation des Solides non cristallins, p. 14, Lyon 1976.

[4] Suchet, J. P., Mat. Res. Bull. 6 (1971) 491.

[5] Clavaguera, N., Clavaguera-Mora, M. T. and CasasVÁzQUEZ, J., J. Non Crystall. Solids 22 (1976) 23. 